JOURNAL OF

FUNCTION SPACES AND APPLICATIONS

Volume 2, Number 2 (2004), 191-215 (c) 2004, Scientific Horizon http://www.jfsa.net

\title{
The Hadamard-Schwarz inequality
}

\section{Tadeusz Iwaniec, Janne Kauhanen, Aleda Kravetz and Chad Scott}

\author{
(Communicated by Carlo Sbordone)
}

2000 Mathematics Subject Classification. 58A10 (Differential forms).

Keywords and phrases. Exterior form, covector, Schwarz inequality, Hadamard inequality.

Abstract. Given $\alpha^{1}, \ldots, \alpha^{k}$ arbitrary exterior forms in $\mathbb{R}^{n}$ of degree $l_{1}, \ldots, l_{k}$, does it follow that

$$
\left|\alpha^{1} \wedge \ldots \wedge \alpha^{k}\right| \leq\left|\alpha^{1}\right| \cdots\left|\alpha^{k}\right|
$$

The answer is no in general. However, it is a persistent, popular and even published misconception that the answer is yes. Of course, a routine calculation reveals that there exists at least a constant $C_{n}$ independent of the forms satisfying

$$
\left|\alpha^{1} \wedge \ldots \wedge \alpha^{k}\right| \leq C_{n}\left|\alpha^{1}\right| \cdots\left|\alpha^{k}\right|
$$

For reasons mentioned in the introduction, we refer to this as the HadamardSchwarz inequality. However, what the best constant is, either overall or for the particular numbers $l_{1}, \ldots, l_{k}$ remains well short of clear.

It is the objective of this paper to explicitly describe the smallest constant for the Hadamard-Schwarz inequality as well as to identify the associated forms for which equality occurs. We have answered these questions for a wide class of integers $0 \leq l_{1}, \ldots, l_{k} \leq n$.

\section{Introduction}

Troughout this paper the Euclidean space $\mathbb{R}^{n}, n \geq 2$, will be equipped with the standard oriented basis $\left\{e_{1}, \ldots, e_{n}\right\}$ and the inner product $\langle\cdot, \cdot\rangle$. 
Let $\bigwedge^{l}=\bigwedge^{l}\left(\mathbb{R}^{n}\right), l=0,1, \ldots, n$, denote the space of exterior forms of degree $l$, also called the $l$-covectors. Thus, $\bigwedge^{0}=\mathbb{R}$ while $\bigwedge^{1}=\bigwedge^{1}\left(\mathbb{R}^{n}\right)$ is just the dual space to $\mathbb{R}^{n}$ of linear forms, simply called covectors. To each vector $v \in \mathbb{R}^{n}$ there corresponds a covector $v^{\prime} \in \Lambda^{1}\left(\mathbb{R}^{n}\right)$ defined by $v^{\prime}(x)=\langle v, x\rangle$ for $x \in \mathbb{R}^{n}$. The dual basis $\left\{e_{1}^{\prime}, \ldots, e_{n}^{\prime}\right\}$ for $\bigwedge^{1}\left(\mathbb{R}^{n}\right)$ will be used to define standard basis for $\bigwedge^{l}\left(\mathbb{R}^{n}\right)$. First recall the wedge product $\alpha \wedge \beta \in \bigwedge^{p+q}$ of $\alpha \in \bigwedge^{p}$ and $\beta \in \bigwedge^{q}$ and note that the exterior multiplication is associative. The standard basis for $\bigwedge^{l}\left(\mathbb{R}^{n}\right), l=1,2, \ldots, n$, consists of prime $l$-covectors

$$
e^{I}=e_{i_{1}}^{\prime} \wedge \ldots \wedge e_{i_{l}}^{\prime}
$$

corresponding to all ordered $l$-tuples $I=\left(i_{1}, \ldots, i_{l}\right), 1 \leq i_{1}<\cdots<i_{l} \leq n$. The usual inner product structure in $\bigwedge^{l}\left(\mathbb{R}^{n}\right)$ is defined once we declare $\left\{e^{I}\right\}$ to be an orthonormal basis. We continue to write $\langle\cdot, \cdot\rangle$ and $|\cdot|$ for this inner product and the induced norm in $\bigwedge^{l}\left(\mathbb{R}^{n}\right)$.

Now, the well know Hadamard inequality for determinants can be written as

$$
\left|\alpha^{1} \wedge \ldots \wedge \alpha^{n}\right| \leq\left|\alpha^{1}\right| \cdots\left|\alpha^{n}\right|
$$

for arbitrary linear forms $\alpha^{1}, \ldots, \alpha^{n} \in \bigwedge^{1}\left(\mathbb{R}^{n}\right)$, see remarks after Corollary 4.1.

On the other hand, the inner product together with the fixed volume element

$$
\mathrm{vol}=e_{1}^{\prime} \wedge \ldots \wedge e_{n}^{\prime} \in \bigwedge\left(\mathbb{R}^{n}\right)
$$

gives rise to the Hodge star duality operator $*: \bigwedge^{l}\left(\mathbb{R}^{n}\right) \rightarrow \bigwedge^{n-l}\left(\mathbb{R}^{n}\right)$, uniquely determined by the equations

$$
\begin{aligned}
* 1 & =\text { vol } \\
\alpha \wedge * \beta & =\langle\alpha, \beta\rangle \text { vol, } \quad \alpha, \beta \in \bigwedge^{l}\left(\mathbb{R}^{n}\right)
\end{aligned}
$$

We emphasize that $*$ is an isometry and $* *=(-1)^{n l+l}: \bigwedge^{l}\left(\mathbb{R}^{n}\right) \rightarrow \bigwedge^{l}\left(\mathbb{R}^{n}\right)$. Therefore, Schwarz inequality for the inner product in $\bigwedge^{l}\left(\mathbb{R}^{n}\right)$ can be stated as

$$
|\alpha \wedge \beta| \leq|\alpha||\beta| \quad \text { for } \alpha \in \bigwedge^{l}\left(\mathbb{R}^{n}\right) \text { and } \beta \in \bigwedge^{n-l}\left(\mathbb{R}^{n}\right)
$$

Since (0.2) includes both (1.1) and (1.2) we refer to it as the HadamardSchwarz inequality.

Definition 1.1. Let $C_{n}\left(l_{1}, \ldots, l_{k}\right)$ denote the smallest constant such that

$$
\left|\alpha^{1} \wedge \ldots \wedge \alpha^{k}\right| \leq C_{n}\left(l_{1}, \ldots, l_{k}\right)\left|\alpha^{1}\right| \cdots\left|\alpha^{k}\right|
$$


for all $\alpha^{i} \in \bigwedge^{l_{i}}\left(\mathbb{R}^{n}\right), i=1, \ldots, k$.

A few results concerning these constants are already known. Indeed, in his now classic [1], H. Federer discusses the issue briefly and presents a few rough bounds. Certainly, $C_{n}\left(l_{1}, \ldots, l_{k}\right)=0$ if $l_{1}+\ldots+l_{k}>n$. Therefore, we always assume, without saying it so every time, that $l_{1}, \ldots, l_{k}$ are nonnegative integers such that

$$
l_{1}+\ldots+l_{k} \leq n
$$

That $C_{n}\left(l_{1}, \ldots, l_{k}\right) \geq 1$ is rather evident. There is also no loss of generality in assuming that $l_{1}+\cdots+l_{k}=n$, which is clear from the formula

$$
C_{n}\left(l_{1}, \ldots, l_{k}\right)=C_{n}\left(l_{1}, \ldots, l_{k}, n-l_{1}-\ldots-l_{k}\right)
$$

see Section 7. Let us begin with an elementary but rather useful result.

Theorem 1.2. Under the assumption $l_{1}+\ldots+l_{k}=n$ inequality $(0.1)$ holds for all forms $\alpha^{1}, \ldots, \alpha^{k}$ if and only if at most two terms in $\left(l_{1}, \ldots, l_{k}\right)$ are greater than 1 .

Therefore, the first opportunity for a constant $C_{n}\left(l_{1}, \ldots, l_{k}\right)$ to be larger than one is in dimension 6 for the case $l_{1}=l_{2}=l_{3}=2$. It is perhaps now possible to see why inequality $(0.1)$ has been mistakenly assumed to be true. Setting $\alpha^{1}=\alpha^{2}=\alpha^{3}=e_{1} \wedge e_{2}+e_{3} \wedge e_{4}+e_{5} \wedge e_{6} \in \Lambda^{2}\left(\mathbb{R}^{6}\right)$ we quickly compute that this is not the case, namely $C_{6}(2,2,2) \geq \frac{2}{\sqrt{3}}>1$. This example is fundamental, for that it leads us to the description of the extremal cases for the inequalities that follow. One of our main results handles $C_{6}(2,2,2)$ and much more.

Theorem 1.3. For $\alpha \in \bigwedge^{2}\left(\mathbb{R}^{n}\right)$ and $\beta \in \bigwedge^{s}\left(\mathbb{R}^{n}\right), s=0,1, \ldots, n$, we have

$$
|\alpha \wedge \beta| \leq C_{n}(2, s)|\alpha||\beta|
$$

where the smallest constant equals

$$
C_{n}(2, s)=\sqrt{\frac{\left[\frac{n-s}{2}\right]\left[\frac{s+2}{2}\right]}{\left[\frac{n-s}{2}\right]+\left[\frac{s}{2}\right]}}
$$

Here, [·] stands for the integer part. Thus, in particular 
Theorem 1.4. For each dimension $n \geq 2$, we have

$$
C_{n}(2,2)=\sqrt{2-2\left[\frac{n}{2}\right]^{-1}}
$$

In view of (1.5) it yields

$$
C_{6}(2,2,2)=\frac{2}{\sqrt{3}}
$$

Repeated application of (1.7) together with analysis of the case of equality occuring in (1.6) leads us to the following.

Theorem 1.5. For $l_{1}=\ldots=l_{k}=2$, with $1 \leq k \leq\left[\frac{n}{2}\right]$, we have

$$
C_{n}\left(l_{1}, \ldots, l_{k}\right)=\prod_{j=1}^{k-1} C_{n}(2,2 j)=\sqrt{\frac{k !\left[\frac{n}{2}\right] !}{\left[\frac{n}{2}\right]^{k}\left[\frac{n}{2}-k\right] !}}
$$

Next recall that the rank of a nonzero $l$-covector $\alpha \in \bigwedge^{l}\left(\mathbb{R}^{n}\right), 1 \leq l \leq n$, is the smallest number of linear forms such that the exterior subalgebra generated by these forms contains $\alpha$. The rank of any 2-covector $\alpha \in$ $\bigwedge^{2}\left(\mathbb{R}^{n}\right)$ is even, say equal to $2 r$. It is not difficult to compute its rank, $r$ is the largest integer such that

$$
\underbrace{\alpha \wedge \cdots \wedge \alpha}_{r \text { times }} \neq 0
$$

More precise variant of the Hadamard-Schwarz inequality takes into account the rank of $\alpha$.

Theorem 1.6. Let $\alpha \in \bigwedge^{2}\left(\mathbb{R}^{n}\right)$ have rank $2 r$ and $\beta$ be arbitrary form of degree $s, 0 \leq s \leq n$. Then

$$
|\alpha \wedge \beta| \leq A_{n, r}(s)|\alpha||\beta|
$$

where the smallest constant is determined by the equation

$$
A_{n, r}^{2}(s)=\max \left\{(l+1)\left(1-\frac{l}{k}\right): 1 \leq k \leq r, s+2 k-n \leq 2 l \leq s\right\}
$$

In general, maximum here occurs when $k=r$. The only exception is the case when $n=2 r$ and at the same time $s$ is odd. In this latter case formula 
(1.12) takes the form

$$
A_{n, r}^{2}(s)=\frac{(s+1)(n-s-1)}{2(n-2)}
$$

If, however, $n \neq 2 r$ or $s$ is even then

$$
\begin{aligned}
& A_{n, r}^{2}(s)= \max \left\{(l+1)\left(1-\frac{l}{r}\right): s+2 r-n \leq 2 l \leq s\right\} \\
&= \begin{cases}\frac{1}{r}\left[\frac{s+2}{2}\right]\left[\frac{2 r-s+1}{2}\right] & \text { if } 0 \leq s<r \\
\frac{1}{r}\left[\frac{r+1}{2}\right]\left[\frac{r+2}{2}\right] & \text { if } r \leq s \leq n-r-2 \\
\frac{1}{r}\left[\frac{n-s}{2}\right]\left[\frac{s+2 r-n+3}{2}\right] & \text { if } n-r-2<s \leq n-2\end{cases}
\end{aligned}
$$

In either case we conclude with a dimension free estimate

$$
A_{n, r}(s) \leq \sqrt{\frac{1}{r}\left[\frac{r+1}{2}\right]\left[\frac{r+2}{2}\right]}
$$

There is a particularly convenient and elegant way of discussing equality occurring in (1.11). For this, we associate with a unit $l$-covector $\alpha \in \bigwedge^{2}\left(\mathbb{R}^{n}\right)$ a linear operator $\Lambda_{\alpha}: \bigwedge^{s}\left(\mathbb{R}^{n}\right) \rightarrow \bigwedge^{s+2}\left(\mathbb{R}^{n}\right), 0 \leq s \leq n$, which carries each element $\beta \in \bigwedge^{s}\left(\mathbb{R}^{n}\right)$ into $\alpha \wedge \beta \in \bigwedge^{s+2}\left(\mathbb{R}^{n}\right)$. Theorems 1.2 and 1.6 provide us with sharp bounds, independent of $\alpha$, for the norms of this operator

$$
\left\|\Lambda_{\alpha}\right\|_{s} \leq C_{n}(2, s)
$$

If we specify the rank of $\alpha$, say $\operatorname{rank} \alpha=2 r$, then

$$
\left\|\Lambda_{\alpha}\right\|_{s} \leq A_{n, r}(s)
$$

The operator $\Lambda_{\alpha}$ also acts on the entire exterior algebra:

$$
\Lambda_{\alpha}: \bigwedge\left(\mathbb{R}^{n}\right) \rightarrow \bigwedge\left(\mathbb{R}^{n}\right)=\bigoplus_{s=0}^{n} \bigwedge^{s}\left(\mathbb{R}^{n}\right)
$$

The following sharp bound for its norm is dimension free:

$$
\left\|\Lambda_{\alpha}\right\| \leq \sqrt{\frac{1}{r}\left[\frac{r+1}{2}\right]\left[\frac{r+2}{2}\right]}
$$

whenever $\alpha$ has rank $2 r$. 
We shall identify all $\alpha$ 's for which equality takes place at (1.16), (1.17) or (1.18). The general name extremal 2-covector will be used for such $\alpha$. Let us state here the result concerning inequality (1.16).

Theorem 1.7. Consider the operator $\Lambda_{\alpha}: \bigwedge^{s}\left(\mathbb{R}^{n}\right) \rightarrow \bigwedge^{s+2}\left(\mathbb{R}^{n}\right)$.

(i) If $s$ equals $n-1$ or $n$, then $\Lambda_{\alpha}$ is the zero operator.

(ii) If $s$ equals 0 or $n-2$, then $\left\|\Lambda_{\alpha}\right\|_{s}=1$ for every unit $\alpha \in \Lambda^{2}\left(\mathbb{R}^{n}\right)$.

(iii) If $s$ equals 1 or $n-3$, then $\left\|\Lambda_{\alpha}\right\|_{s} \leq 1$. Equality occurs if and only if $\operatorname{rank} \alpha<n$.

(iv) If $1<s<n-3$, then $\left\|\Lambda_{\alpha}\right\|_{s} \leq C_{n}(2, s)$. Equality occurs if and only if

$$
\alpha=\frac{1}{\sqrt{r}}\left(\xi^{1} \wedge \zeta^{1}+\ldots+\xi^{r} \wedge \zeta^{r}\right)
$$

where $r=\left[\frac{n-s}{2}\right]+\left[\frac{s}{2}\right]$ and $\left\{\xi^{1}, \zeta^{1}, \ldots, \xi^{r}, \zeta^{r}\right\}$ is any orthonormal set of linear forms.

Once the extremal 2-covector $\alpha$ for the operator $\Lambda_{\alpha}: \Lambda^{s}\left(\mathbb{R}^{n}\right) \rightarrow$ $\Lambda^{s+2}\left(\mathbb{R}^{n}\right)$ is fixed, we can try to identify the associated space of all $\beta \in \Lambda^{s}\left(\mathbb{R}^{n}\right)$ for which $\Lambda_{\alpha}$ attains its norm, that is

$$
E_{\alpha}(s)=\left\{\beta \in \bigwedge^{s}\left(\mathbb{R}^{n}\right):|\alpha \wedge \beta|=C_{n}(2, s)|\beta|\right\}
$$

Note that $C_{n}^{2}(2, s)$ is the largest eigenvalue of the operator $\Lambda_{\alpha}^{*} \Lambda_{\alpha}$ and $E_{\alpha}(s)$ is its eigenspace. Before jumping to any conclusion let us remark that the isomorphism

$$
* \Lambda_{\alpha}: \bigwedge^{s}\left(\mathbb{R}^{n}\right) \rightarrow \bigwedge^{n-s-2}\left(\mathbb{R}^{n}\right)
$$

maps $E_{\alpha}(s)$ onto $E_{\alpha}(n-s-2)$. We infer from this that

$$
A_{n, r}(s)=A_{n, r}(n-s-2)
$$

Hence

$$
C_{n}(2, s)=C_{n}(2, n-s-2)
$$

We are only interested in the cases when these constants are greater than 1 . By Theorem 1.7 this means that we are confined to $1<s<n-3$. Let us close the introduction with another of our main results detailing the extremals. 
Theorem 1.8. Let the extremal $\alpha$ be given by (1.19). Then $E_{\alpha}(s)$ consists of all $\beta \in \Lambda^{s}\left(\mathbb{R}^{n}\right)$ such that

$$
\beta=\underbrace{\alpha \wedge \cdots \wedge \alpha}_{l \text { times }} \wedge \eta, \quad l=\left[\frac{s}{2}\right]
$$

where $\eta$ is any real number if $s$ is even, and $\eta$ is any linear form orthogonal to $\left\{\xi^{1}, \zeta^{1}, \ldots, \xi^{r}, \zeta^{r}\right\}$ if $s$ is odd.

\section{Pullback}

We take the time now to discuss how a change of basis affects the exterior forms. First recall that every linear transformation $A: \mathbb{R}^{n} \rightarrow \mathbb{R}^{n}$ induces the pullback of $l$-covectors, $l=1,2, \ldots, n$, denoted by $A_{\#}: \bigwedge^{l}\left(\mathbb{R}^{n}\right) \rightarrow \bigwedge^{l}\left(\mathbb{R}^{n}\right)$. Precisely, if $\xi \in \Lambda^{l}\left(\mathbb{R}^{n}\right)$ is an alternating $l$-linear function on $\mathbb{R}^{n}$ then for arbitrary vectors $x_{1}, x_{2}, \ldots, x_{l} \in \mathbb{R}^{n}$ we define

$$
\left(A_{\#} \xi\right)\left(x_{1}, \ldots, x_{l}\right)=\xi\left(A x_{1}, \ldots, A x_{l}\right)
$$

Pulling back has many nice properties. Among them, we have

$$
\begin{aligned}
\left(A_{\#} \xi\right) \wedge\left(A_{\#} \zeta\right) & =A_{\#}(\xi \wedge \zeta) \\
\left(A^{-1}\right)_{\#} & =\left(A_{\#}\right)^{-1} \\
\left(A^{t}\right)_{\#} & =\left(A_{\#}\right)^{t} \\
(A B)_{\#} & =B_{\#} A_{\#}
\end{aligned}
$$

Let $\mathcal{O}\left(\mathbb{R}^{n}\right)$ denote the orthogonal group of $\mathbb{R}^{n}$.

Definition 2.1. We call

$$
\mathcal{O}_{\#}\left(\bigwedge^{l}\left(\mathbb{R}^{n}\right)\right)=\left\{A_{\#}: \bigwedge^{l}\left(\mathbb{R}^{n}\right) \rightarrow \bigwedge^{l}\left(\mathbb{R}^{n}\right) \mid A \in \mathcal{O}\left(\mathbb{R}^{n}\right)\right\}
$$

the induced orthogonal subgroup of $\mathcal{O}\left(\bigwedge^{l}\left(\mathbb{R}^{n}\right)\right)$.

It follows from the identities above that $\mathcal{O}_{\#}\left(\bigwedge^{l}\left(\mathbb{R}^{n}\right)\right)$ is indeed a subgroup of the orthogonal group $\mathcal{O}\left(\bigwedge^{l}\left(\mathbb{R}^{n}\right)\right)$ in the space $\bigwedge^{l}\left(\mathbb{R}^{n}\right)$. Perhaps more important is that the Hadamard-Schwarz inequality is invariant under induced orthogonal transformations. Thus, in particular, $\Lambda_{\alpha}$ and $\Lambda_{A_{\#} \alpha}$ have the same norms. If $\alpha$ is an extremal 2-covector so is $A_{\#} \alpha$. One of the first things we must investigate in this connection is the size of the induced subgroup in the space $\bigwedge^{2}\left(\mathbb{R}^{n}\right)$.

The full orthogonal group $\mathcal{O}\left(\bigwedge^{2}\left(\mathbb{R}^{n}\right)\right)$ is a Lie group of dimension $\frac{1}{2}\left(\begin{array}{l}n \\ 2\end{array}\right)\left[\left(\begin{array}{l}n \\ 2\end{array}\right)-1\right]=\frac{1}{8}(n-2)(n-1) n(n+1)$. Observe that $\operatorname{dim} \mathcal{O}_{\#}\left(\bigwedge^{2}\left(\mathbb{R}^{n}\right)\right)=$ 
$\left.\operatorname{dim} \mathcal{O}\left(\mathbb{R}^{n}\right)\right)=\left(\begin{array}{l}n \\ 2\end{array}\right)$. Incidentally, this is precisely the dimension of the linear space $\Lambda^{2}\left(\mathbb{R}^{n}\right)$. In a very simplistic way, we can try to use an induced orthogonal transformation to rotate $\alpha \in \Lambda^{2}\left(\mathbb{R}^{n}\right)$ into a suitable 2-covector of the same length. Unfortunately, in spite of this promising analysis of dimensions, the induced subgroup $\mathcal{O}_{\#}\left(\bigwedge^{2}\left(\mathbb{R}^{n}\right)\right)$ still does not act transitively; elements of an orbit have the same rank. The following result comes to the rescue.

Theorem 2.2. Let $\alpha \in \bigwedge^{2}\left(\mathbb{R}^{n}\right)$ have rank $2 r$. Then there exists $A \in \mathcal{O}\left(\mathbb{R}^{n}\right)$ such that

$$
A_{\#} \alpha=a_{1} e_{1}^{\prime} \wedge e_{2}^{\prime}+\ldots+a_{r} e_{2 r-1}^{\prime} \wedge e_{2 r}^{\prime}
$$

for some positive numbers $a_{1}, \ldots, a_{r}$.

Here is another way of stating this fact. Given $\alpha \in \bigwedge^{2}\left(\mathbb{R}^{n}\right)$ of rank $2 r$, there exists an orthonormal basis in $\bigwedge^{1}\left(\mathbb{R}^{n}\right)$, say

$$
\left\{\xi^{1}, \zeta^{1}, \ldots, \xi^{r}, \zeta^{r}, \ldots\right\}
$$

such that $\alpha$ can be expressed as

$$
\alpha=a_{1}\left(\xi^{1} \wedge \zeta^{1}\right)+\ldots+a_{r}\left(\xi^{r} \wedge \zeta^{r}\right)
$$

where $a_{1}, \ldots, a_{r}$ are positive numbers. Here and in the sequel we have adopted the convention that the sequence of covectors at (2.5) ends once it reaches $n$ terms, which can be either even or odd number. We call (2.5) an associated basis for $\alpha$. Different associated bases yield the same number $r$, that is, half of the rank of $\alpha$.

A few words should be said about this result. While 2-covectors had been discussed in the context of simplectic geometry, the fact that one can find an orthonormal transformation, to represent them in the form (2.6), seems to be unfamiliar. We include a proof of this fact as it might be of independent interest. Theorem 2.2 provides us with a powerful tool for establishing sharp bounds involving forms of degree 2 .

One of the major difficulties in dealing with forms of higher degree is that the induced group $\mathcal{O}_{\#}\left(\bigwedge^{l}\left(\mathbb{R}^{n}\right)\right)$ for $l>2$ is too small. In quest of the best constant $C_{n}\left(l_{1}, \ldots, l_{k}\right)$ for such forms, we are faced with fascinating questions regarding other possible orthogonal subgroups of $\mathcal{O}\left(\bigwedge^{l}\left(\mathbb{R}^{n}\right)\right)$. But, we shall not enter into these quite involved problem.

\section{Proof of Theorem 2.2}

We'll split this proof up into a short sequence of lemmas. 
Lemma 3.1. Let $V$ be an inner product space of dimension $n \geq 2$ and let $\phi \in \Lambda^{2}(V)$ be a nonzero exterior form of degree 2. Then there exist orthonormal vectors $u, v \in V$ such that

$$
\phi(u, v)=-\phi(v, u) \neq 0
$$

Furthermore, the linear space

$$
W=\{x \in V: \phi(u, x)=\phi(v, x)=0\}
$$

is orthogonal to $u$ and $v$.

Proof. By the Riesz Representation Theorem, any 2-form on $V$ uniquely determines a linear transformation $L: V \rightarrow V$ by the rule

$$
\phi(u, v)=\langle L u, v\rangle \quad \text { for } \quad u, v \in V
$$

Also note that it is possible to make $V \times V$ into an inner product space. Indeed, we simply define

$$
\left\langle\left(u_{1}, u_{2}\right) \mid\left(v_{1}, v_{2}\right)\right\rangle=\left\langle u_{1}, v_{1}\right\rangle+\left\langle u_{2}, v_{2}\right\rangle
$$

Next, we define a linear transformation

$$
L: V \times V \rightarrow V \times V
$$

by setting

$$
L(x, y)=(L y,-L x)
$$

This linear map is symmetric. Indeed

$$
\begin{aligned}
\langle L(x, y) \mid(u, v)\rangle & =\langle(L y,-L x) \mid(u, v)\rangle \\
& =\langle L y, u\rangle+\langle-L x, v\rangle \\
& =-\langle y, L u\rangle+\langle x, L v\rangle \\
& =\langle x, L v\rangle+\langle y,-L u\rangle \\
& =\langle(x, y) \mid(L v,-L u)\rangle \\
& =\langle(x, y) \mid L(u, v)\rangle
\end{aligned}
$$

as desired. Accordingly, all the eigenvalues of $L$ are real numbers and at least one of them, denoted by $\lambda$, is different from zero. Otherwise, $L$ would be the zero transformation. Let $(u, v) \in V \times V$ be a nonzero eigenvector 
corresponding to $\lambda$, say

$$
L(u, v)=\lambda(u, v), \quad|u|^{2}+|v|^{2}=2
$$

or equivalently

$$
\begin{aligned}
& L v=\lambda u \\
& L u=-\lambda v
\end{aligned}
$$

Note that $u$ and $v$ are orthogonal since $\lambda\langle u, v\rangle=\langle L v, v\rangle=\phi(v, v)=0$. We also find that $|u|=|v|=1$ and

$$
\begin{aligned}
2 \phi(u, v) & =\phi(u, v)-\phi(v, u) \\
& =\langle L u, v\rangle-\langle L v, u\rangle \\
& =-\lambda\langle v, v\rangle-\lambda\langle u, u\rangle \\
& =-\lambda\left(|u|^{2}+|v|^{2}\right) \neq 0
\end{aligned}
$$

To complete the proof of the lemma, consider an arbitrary $x \in W$. That is, $\phi(u, x)=\phi(v, x)=0$. By (3.3), we obtain $\langle L u, x\rangle=\langle L v, x\rangle=0$ and by (3.8) it follows that $\langle v, x\rangle=\langle u, x\rangle=0$, as desired.

Lemma 3.2. Let $V$ be an inner product space of dimension $n \geq 2$ and let $\phi \in \Lambda^{2}(V)$ be a nonzero exterior 2-form on $V$. Then, there exists an orthonormal basis for $V$, denoted by

$$
\left\{u_{1}, v_{1}, u_{2}, v_{2}, u_{3}, v_{3}, \ldots, u_{s}, v_{s}, \ldots\right\}
$$

such that for some integer $1 \leq s \leq \frac{n}{2}$, we have

$$
\left\{\begin{aligned}
\phi\left(u_{1}, v_{1}\right) & =-\phi\left(v_{1}, u_{1}\right) \neq 0 \\
\phi\left(u_{2}, v_{2}\right) & =-\phi\left(v_{2}, u_{2}\right) \neq 0 \\
\vdots & \\
\phi\left(u_{s}, v_{s}\right) & =-\phi\left(v_{s}, u_{s}\right) \neq 0
\end{aligned}\right.
$$

and

$$
\phi(x, y)=0
$$

for all other possible pairs $(x, y)$ of the basis vectors.

Remark. The number $2 s$ in (3.10) is exactly equal to the rank of $\xi$. Hence, in particular, the rank of a 2 -form is always even. It does not depend on the particular choice of the basis stated in this lemma. Note also that a 
2 -form $\xi \in \bigwedge^{2}\left(\mathbb{R}^{n}\right)$ has rank $2 s$ if and only if

$$
\underbrace{\xi \wedge \xi \wedge \ldots \wedge \xi}_{s \text { times }} \neq 0 \text { and } \underbrace{\xi \wedge \xi \wedge \ldots \wedge \xi}_{s+1 \text { times }} \neq 0
$$

Proof. The proof uses an induction argument by repeated application of Lemma 3.1. Let $V_{1}=V$. By virtue of Lemma 3.1, we can pick up two orthonormal vectors $u_{1}, v_{1} \in V_{1}$ such that

$$
\phi\left(u_{1}, v_{1}\right)=-\phi\left(v_{1}, u_{1}\right) \neq 0
$$

and the space $V_{2}=\left\{x \in V: \phi\left(u_{1}, x\right)=0=\phi\left(v_{1}, x\right)=0\right\}$ is orthogonal to $u_{1}$ and $v_{1}$. Certainly, $\operatorname{dim} V_{2}=n-2$. We look at $\phi$ as a 2 -form on $V_{2}$. If $\phi$ restricted to $V_{2}$ vanishes identically (i.e. $\phi(x, y)=0$ for all $x, y \in V_{2}$ ) then we stop the induction. The basis $\left\{u_{1}, v_{1}, u_{2}, v_{2}, \ldots\right\}$ is then constructed by taking $\left\{u_{2}, v_{2}, \ldots\right\}$, an arbitrary orthonormal basis for $V_{2}$. If $\phi$ restricted to $V_{2}$ is not a zero 2 -form, we apply Lemma 3.1 again to construct a pair $u_{2}, v_{2} \in V_{2}$ of orthonormal vectors such that

$$
\phi\left(u_{2}, v_{2}\right)=-\phi\left(v_{2}, u_{2}\right) \neq 0
$$

Moreover, the space

$$
V_{3}=\left\{x \in V_{2}: \phi\left(u_{2}, x\right)=\phi\left(v_{2}, x\right)=0\right\}
$$

is orthogonal to $u_{1}, v_{1}, u_{2}$ and $v_{2}$. We stop the construction if $\phi$ restricted to $V_{3}$ is the zero form. If not, we proceed by induction obtaining the space

$$
V_{s+1}=\left\{x \in V_{s}: \phi\left(u_{s}, x\right)=\phi\left(v_{s}, x\right)=0\right\}, \quad 2 s \leq n
$$

which is orthogonal to $u_{1}, v_{1}, \ldots, u_{s}$ and $v_{s}$ and such that $\phi$ restricted to $V_{s+1}$ is the zero form. The desired sequence (3.9) is then obtained by adding to $\left\{u_{1}, v_{1}, \ldots, u_{s}, v_{s}\right\}$ any orthonormal basis for $V_{s+1}$.

Lemma 3.3. Given $\phi \in \bigwedge^{2}\left(\mathbb{R}^{n}\right)$, let $\left\{u_{1}^{\prime}, v_{1}^{\prime}, u_{2}^{\prime}, v_{2}^{\prime}, \ldots, u_{s}^{\prime}, v_{s}^{\prime}, \ldots\right\}$ denote the dual basis for $\bigwedge^{1}\left(\mathbb{R}^{n}\right)$ obtained from (3.9) with $V=\mathbb{R}^{n}$. Then

$$
\phi=\lambda_{1}\left(u_{1}^{\prime} \wedge v_{1}^{\prime}\right)+\lambda_{2}\left(u_{2}^{\prime} \wedge v_{2}^{\prime}\right)+\cdots+\lambda_{s}\left(u_{s}^{\prime} \wedge v_{s}^{\prime}\right)
$$

where $\lambda_{i}=\phi\left(u_{i}, v_{i}\right)$ for $i=1,2, \ldots, s$. 
Proof. Consider two arbitrary vectors in $\mathbb{R}^{n}$

$$
\begin{aligned}
& x=\sum_{i}\left(\alpha_{i} u_{i}+\beta_{i} v_{i}\right) \\
& y=\sum_{j}\left(\gamma_{j} u_{j}+\delta_{j} v_{j}\right)
\end{aligned}
$$

The bilinearity of $\phi$ and $\phi\left(u_{i}, u_{j}\right)=\phi\left(v_{i}, v_{j}\right)=0$ (Lemma 3.2) allow us to compute

$$
\begin{aligned}
\phi(x, y) & =\sum_{i, j}\left\{\phi\left(\alpha_{i} u_{i}, \gamma_{j} u_{j}\right)+\phi\left(\alpha_{i} u_{i}, \delta_{j} v_{j}\right)+\phi\left(\beta_{i} v_{i}, \gamma_{j} u_{j}\right)+\phi\left(\beta_{i} v_{i}, \delta_{j} v_{j}\right)\right\} \\
& =\sum_{i, j}\left(\alpha_{i} \delta_{j}-\beta_{i} \gamma_{j}\right) \phi\left(u_{i}, v_{j}\right)
\end{aligned}
$$

Also, $\phi\left(u_{i}, v_{j}\right)=0$ whenever $i \neq j$ which simplifies this expression to

$$
\phi(x, y)=\sum_{i}\left(\alpha_{i} \delta_{i}-\beta_{i} \gamma_{i}\right) \phi\left(u_{i}, v_{i}\right)
$$

On the other hand, by the definition of the wedge product, the 2-form $u_{i}^{\prime} \wedge v_{i}^{\prime}$ acts on $(x, y)$ as

$$
\left(u_{i}^{\prime} \wedge v_{i}^{\prime}\right)(x, y)=u_{i}^{\prime}(x) v_{i}^{\prime}(y)-u_{i}^{\prime}(y) v_{i}^{\prime}(x)=\alpha_{i} \delta_{i}-\gamma_{i} \beta_{i}
$$

which is clear from the definition of the dual basis. In other words

$$
\phi=\sum_{i}\left(u_{i}^{\prime} \wedge v_{i}^{\prime}\right) \phi\left(u_{i}, v_{i}\right)=\lambda_{1}\left(u_{1}^{\prime} \wedge v_{1}^{\prime}\right)+\cdots+\lambda_{s}\left(u_{s}^{\prime} \wedge v_{s}^{\prime}\right)
$$

as desired.

To finally settle Theorem 2.2, we select an orthogonal transformation $A \in \mathcal{O}\left(\mathbb{R}^{n}\right)$ such that

$$
\begin{cases}A u_{i}=e_{2 i-1} & i=1, \ldots, k \quad(\text { or } k+1 \text { if } n=2 k+1) \\ A v_{i}=e_{2 i} & i=1, \ldots, k\end{cases}
$$

And so we have

$$
A_{\#} \phi=\lambda_{1}\left(e_{1}^{\prime} \wedge e_{2}^{\prime}\right)+\cdots+\lambda_{k}\left(e_{2 k-1}^{\prime} \wedge e_{2 k}^{\prime}\right), \quad \lambda_{s+1}=\lambda_{s}=0
$$




\section{Proof of Theorem 1.2}

Let us begin with some general considerations. Using the standard basis $\left\{e_{1}^{\prime}, \ldots, e_{n}^{\prime}\right\}$ for the space of linear forms, we can express any $s$-covector as

$$
\beta=\sum_{1 \leq i_{1}<\ldots<i_{s} \leq n} b_{i_{1}, \ldots, i_{s}} e_{i_{1}}^{\prime} \wedge \cdots \wedge e_{i_{s}}^{\prime}, \quad|\beta|^{2}=\sum_{1 \leq i_{1}<\ldots<i_{s} \leq n} b_{i_{1}, \ldots, i_{s}}^{2}
$$

For $\alpha=e_{1}^{\prime}$ we see that

$$
|\alpha \wedge \beta|^{2}=\sum_{2 \leq i_{1}<\ldots<i_{s} \leq n} b_{i_{1}, \ldots, i_{s}}^{2} \leq|\beta|^{2}=|\alpha|^{2}|\beta|^{2}
$$

Equality holds if and only if the coefficients $b_{1, i_{2}, \ldots, i_{s}}$ vanish. It is important to realize that the induced orthogonal subgroup $\mathcal{O}_{\#}\left(\bigwedge^{1}\left(\mathbb{R}^{n}\right)\right)$ actually coincides with the full group $\mathcal{O}\left(\bigwedge^{1}\left(\mathbb{R}^{n}\right)\right)$. Therefore, each unit covector $\alpha \in \bigwedge^{l}\left(\mathbb{R}^{n}\right)$ can be rotated to $e_{1}^{\prime}$ by the dual of an element $A \in \mathcal{O}\left(\mathbb{R}^{n}\right)$. As pulling back preserves orthogonality on each level of the degree of forms we have

$$
|\alpha \wedge \beta|=\left|A_{\#}(\alpha \wedge \beta)\right|=\left|A_{\#} \alpha \wedge A_{\#} \beta\right|=\left|e_{1}^{\prime} \wedge A_{\#} \beta\right| \leq\left|A_{\#} \beta\right|=|\alpha||\beta|
$$

Equivalently, one can view $\alpha$ as element of an orthonormal basis for the space $\Lambda^{1}\left(\mathbb{R}^{n}\right)$ and then express $\beta$ in terms of this basis. In much the same way as for the standard basis we conclude with the following.

Corollary 4.1. If $\alpha \in \bigwedge^{1}\left(\mathbb{R}^{n}\right)$ and $\beta \in \bigwedge^{s}\left(\mathbb{R}^{n}\right), 0 \leq s \leq n$, then

$$
|\alpha \wedge \beta| \leq|\alpha||\beta|
$$

We have equality if and only if $\beta$ belongs to the subalgebra generated by the covectors orthogonal to $\alpha$.

Repeated application of this result yields Hadamard's inequality (1.1). We also see that equality occurs at (1.1) if and only if the covectors $\alpha^{1}, \ldots, \alpha^{n}$ are mutually orthogonal. Having disposed of this corollary we proceed to the proof of Theorem 1.2.

Proof of Theorem 1.2. First assume that at most two of the forms $\alpha^{1}, \ldots, \alpha^{k}$ have degree greater than one. Since $l_{1}+\ldots+l_{k}=n$ we can use Schwarz inequality (1.2) to write

$$
\left|\alpha^{1} \wedge \ldots \wedge \alpha^{k}\right|=\left|\left(\alpha^{1} \wedge \ldots \wedge \alpha^{i}\right) \wedge\left(\alpha^{i+1} \wedge \ldots \wedge \alpha^{k}\right)\right| \leq\left|\alpha^{1} \wedge \ldots \wedge \alpha^{i}\right|\left|\alpha^{i+1} \wedge \ldots \wedge \alpha^{k}\right|
$$

Here we have split the wedge product in such a way that the forms of degree greater than one are in different groups. By Corollary 4.1 we then conclude 
with the inequality

$$
\left|\alpha^{1} \wedge \ldots \wedge \alpha^{k}\right| \leq\left|\alpha^{1}\right| \cdots\left|\alpha^{k}\right|
$$

Suppose now that at least three terms in the sequence $\left(l_{1}, \ldots, l_{k}\right)$, say $l_{1}, l_{2}$ and $l_{3}$, are greater than one. We shall see that (4.2) fails for some covectors. Recall the notation

$$
e^{I}=e_{i_{1}}^{\prime} \wedge \ldots \wedge e_{i_{l}}^{\prime}, \quad I=\left(i_{1}, \ldots, i_{l}\right) \quad \text { with } \quad 1 \leq i_{1}<\cdots<i_{l} \leq n
$$

where we put $e^{I}=1$ if $I$ is empty. Next, consider the following forms

$$
\begin{aligned}
\omega & =e_{1}^{\prime} \wedge e_{2}^{\prime}+e_{3}^{\prime} \wedge e_{4}^{\prime}+e_{5}^{\prime} \wedge e_{6}^{\prime} \\
\alpha^{1} & =\omega \wedge e^{I_{1}}, \quad \alpha^{2}=\omega \wedge e^{I_{2}}, \quad \alpha^{3}=\omega \wedge e^{I_{3}}, \quad \alpha^{4}=e^{I_{4}}, \ldots, \alpha^{k}=e^{I_{k}}
\end{aligned}
$$

where $I_{1}, \ldots, I_{k}$ are disjoint subsets of $\{7,8, \ldots, n\}$ whose cardinality equals $l_{1}-2, l_{2}-2, l_{3}-2, l_{4}, \ldots, l_{k}$, respectively. We find that

$$
\left|\alpha^{1} \wedge \ldots \wedge \alpha^{k}\right|=|\omega \wedge \omega \wedge \omega|=\frac{2}{\sqrt{3}}|\omega||\omega||\omega|=\frac{2}{\sqrt{3}}\left|\alpha^{1}\right| \cdots\left|\alpha^{k}\right|
$$

completing the proof of Theorem 1.2.

Remark. It follows from the above arguments that the smallest value of the constants $C_{n}\left(l_{1}, \ldots, l_{k}\right)$, next to 1 , is $\frac{2}{\sqrt{3}}$.

\section{Proof of Theorem 1.4}

Although Theorem 1.4 is only a special case of Theorem 1.3 it is advantagous to prove Theorem 1.4 first, as it might clarify the forthcoming computations.

We shall show more than (1.8), namely

$$
|\xi \wedge \zeta| \leq \sqrt{2-\frac{2}{k}}|\xi||\zeta|
$$

whenever $\xi, \zeta \in \Lambda^{2}\left(\mathbb{R}^{n}\right)$ and $\operatorname{rank} \xi=2 k, 2 \leq k \leq\left[\frac{n}{2}\right]$. Thus, there exists an orthonormal basis $p^{1}, q^{1}, p^{2}, q^{2}, \ldots$ for $\bigwedge^{1}\left(\mathbb{R}^{n}\right)$ such that

$$
\xi=a_{1} p^{1} \wedge q^{1}+\cdots+a_{k} p^{k} \wedge q^{k}
$$

where $a_{1}, \ldots, a_{k}>0$. First assume that $\zeta$ has similar form:

$$
\zeta=b_{1} p^{1} \wedge q^{1}+\cdots+b_{k} p^{k} \wedge q^{k}
$$


It follows easily that

$$
\xi \wedge \zeta=\sum_{1 \leq i<j \leq k}\left(a_{i} b_{j}+a_{j} b_{i}\right) p^{i} \wedge q^{i} \wedge p^{j} \wedge q^{j}
$$

By orthogonality, we compute

$$
|\xi \wedge \zeta|^{2}=\sum_{1 \leq i<j \leq k}\left(a_{i} b_{j}+a_{j} b_{i}\right)^{2}
$$

On the other hand, $|\xi|^{2}=a_{1}^{2}+\cdots+a_{k}^{2}$ and $|\zeta|^{2}=b_{1}^{2}+\cdots+b_{k}^{2}$. Thus,

$$
\begin{aligned}
|\xi|^{2}|\zeta|^{2} & =\sum_{1 \leq i, j \leq k} a_{i}^{2} b_{j}^{2} \\
& =\sum_{1 \leq i<j \leq k}\left(a_{i}^{2} b_{j}^{2}+a_{j}^{2} b_{i}^{2}\right)+\frac{1}{k-1} \sum_{1 \leq i<j \leq k}\left(a_{i}^{2} b_{i}^{2}+a_{j}^{2} b_{j}^{2}\right)
\end{aligned}
$$

as is easy to verify. Inequality (5.1) reads as

$$
\begin{gathered}
(2 k-2) \sum_{1 \leq i<j \leq k}\left(a_{i}^{2} b_{j}^{2}+a_{j}^{2} b_{i}^{2}\right)+2 \sum_{1 \leq i<j \leq k}\left(a_{i}^{2} b_{i}^{2}+a_{j}^{2} b_{j}^{2}\right) \\
\geq k \sum_{1 \leq i<j \leq k}\left(a_{i}^{2} b_{j}^{2}+a_{j}^{2} b_{i}^{2}+2 a_{i} a_{j} b_{i} b_{j}\right)
\end{gathered}
$$

It is a simple matter of comparing the terms to notice that this inequality takes the form

$$
(k-2) \sum_{1 \leq i<j \leq k}\left(a_{i} b_{j}-a_{j} b_{i}\right)^{2}+2 \sum_{1 \leq i<j \leq k}\left(a_{i} b_{i}-a_{j} b_{j}\right)^{2} \geq 0
$$

which is always true. Now, we address the question of when equality occurs. Consider two sets of coefficients in (5.2) and (5.3), $a=\left(a_{1}, \ldots, a_{k}\right) \in \mathbb{R}^{k}$ and $b=\left(b_{1}, \ldots, b_{k}\right) \in \mathbb{R}^{k}$. We can certainly restrict ourselves to nonzero vector $b \in \mathbb{R}^{k}$, since otherwise equality is evident. If $k=2$ we have one system of equations

$$
a_{i} b_{i}-a_{j} b_{j}=0
$$

for $1 \leq i<j \leq 2$. This simply means that $b=\left(\lambda / a_{1}, \lambda / a_{2}\right)$ with some $\lambda \neq 0$. For $k>2$, however, we have two systems of equations for the coordinates of the vectors $a, b \in \mathbb{R}^{k}$ :

$$
a_{i} b_{j}-a_{j} b_{i}=0 \quad \text { and } \quad a_{i} b_{i}-a_{j} b_{j}=0
$$


for $1 \leq i<j \leq k$. The first system means exactly that $b=\lambda a$ with some $\lambda \neq 0$. The second system yields $a_{1}^{2}=a_{2}^{2}=\cdots=a_{k}^{2}$. Summarizing, since $a_{1}, \ldots, a_{k}$ are positive we find that

$$
\xi=\alpha\left(p^{1} \wedge q^{1}+\cdots+p^{k} \wedge q^{k}\right) \quad \text { and } \quad \zeta=\beta\left(p^{1} \wedge q^{1}+\cdots+p^{k} \wedge q^{k}\right)
$$

for some $\alpha, \beta \in \mathbb{R}$.

Next, we relax the assumption that $\zeta$ takes the form (5.3). While keeping $\xi$ the same, we now write $\zeta=\zeta_{0}+\epsilon \in \Lambda^{2}\left(\mathbb{R}^{n}\right)$, where

$$
\zeta_{0}=b_{1} p^{1} \wedge q^{1}+\cdots+b_{k} p^{k} \wedge q^{k}
$$

and

$$
\epsilon=\sum_{i \neq j} \alpha_{i j} p^{i} \wedge q^{j}+\sum_{i<j} \beta_{i j} p^{i} \wedge q^{j}+\sum_{i<j} \gamma_{i j} q^{i} \wedge q^{j}=\alpha+\beta+\gamma
$$

Let us emphasise explicitely that the basis covectors which are different from $\left\{p^{1}, q^{1}, \ldots, p^{k}, q^{k}\right\}$ are also present in (5.10), although they play no essential role there. Now, by orthogonality we have

$$
|\zeta|^{2}=\left|\zeta_{0}\right|^{2}+|\epsilon|^{2}=\left|\zeta_{0}\right|^{2}+|\alpha|^{2}+|\beta|^{2}+|\gamma|^{2}
$$

We also have

$$
\xi \wedge \zeta=\xi \wedge \zeta_{0}+\xi \wedge \alpha+\xi \wedge \beta+\xi \wedge \gamma
$$

It is helpful to note that all of the terms in the right hand side of this equation are orthogonal. To see this, we write

$$
\begin{aligned}
\xi \wedge \zeta_{0} & =\sum_{1 \leq i<j \leq k}\left(a_{i} b_{j}+a_{j} b_{i}\right) p^{i} \wedge q^{i} \wedge p^{j} \wedge q^{j} \\
\xi \wedge \alpha & =\sum_{l=1}^{k} \sum_{i \neq j} \alpha_{i j} a_{l} p^{l} \wedge q^{l} \wedge p^{i} \wedge q^{j} \\
\xi \wedge \beta & =\sum_{l=1}^{k} \sum_{i<j} \beta_{i j} a_{l} p^{l} \wedge q^{l} \wedge p^{i} \wedge p^{j} \\
\xi \wedge \gamma & =\sum_{l=1}^{k} \sum_{i<j} \gamma_{i j} a_{l} p^{l} \wedge q^{l} \wedge q^{i} \wedge q^{j}
\end{aligned}
$$

Notice that none of the wedge products appearing in any one of these equations appears in any of the other three. These wedge products form an 
orthonormal basis for $\bigwedge^{4}\left(\mathbb{R}^{n}\right)$, thus

$$
|\xi \wedge \zeta|^{2}=\left|\xi \wedge \zeta_{0}\right|^{2}+|\xi \wedge \alpha|^{2}+|\xi \wedge \beta|^{2}+|\xi \wedge \gamma|^{2}
$$

We have already established the inequality

$$
\left|\xi \wedge \zeta_{0}\right|^{2} \leq 2\left(1-\frac{1}{k}\right)|\xi|^{2}\left|\zeta_{0}\right|^{2}
$$

As can be seen by looking at (5.15) and (5.16) above, there is no repetition of terms and consequently orthogonality gives

$$
|\xi \wedge \beta|^{2}=\sum_{l=1}^{k} \sum_{i<j} \beta_{i j}^{2} a_{l}^{2}=\left(\sum_{l=1}^{k} a_{l}^{2}\right)\left(\sum_{i<j} \beta_{i j}^{2}\right)=|\xi|^{2}|\beta|^{2}
$$

and similarly

$$
|\xi \wedge \gamma|^{2}=|\xi|^{2}|\gamma|^{2}
$$

To effectively handle the term $\xi \wedge \alpha$, we notice that the sum in (5.14) actually runs over those indices for which $l \neq i \neq j \neq l$. The other terms simply vanish. Restricted to these indices, we see that there is no repetition of terms and, as before, we can write

$$
|\xi \wedge \alpha|^{2}=\sum_{l \neq i \neq j \neq l} \alpha_{i j}^{2} a_{l}^{2} \leq \sum_{l=1}^{k} \sum_{i \neq j} \alpha_{i j}^{2} a_{l}^{2}=|\xi|^{2}|\alpha|^{2}
$$

Finally, we see that (5.19), (5.20) and (5.21) yield

$$
|\xi \wedge \epsilon|^{2} \leq|\xi|^{2}|\epsilon|^{2}
$$

Combining this with (5.18) gives

$$
\begin{aligned}
|\xi \wedge \zeta|^{2} & =\left|\xi \wedge \zeta_{0}\right|^{2}+|\xi \wedge \epsilon|^{2} \\
& =2\left(1-\frac{1}{k}\right)|\xi|^{2}\left|\zeta_{0}\right|^{2}+|\xi|^{2}|\epsilon|^{2} \\
& \leq 2\left(1-\frac{1}{k}\right)|\xi|^{2}\left(\left|\zeta_{0}\right|^{2}+|\epsilon|^{2}\right) \\
& =2\left(1-\frac{1}{k}\right)|\xi|^{2}|\zeta|^{2}
\end{aligned}
$$

as desired. 
We notice here that if the constant $2\left(1-\frac{1}{k}\right)$ is bigger than 1 then $k \geq 3$ and this proof reveals that for equality to hold in (5.23), we need $|\xi|^{2}|\epsilon|^{2}=0$ $\left(2-\frac{2}{k}\right.$ is strictly greater than 1 for $\left.k \geq 3\right)$. But this leaves only two possibilities: $\xi=0$ or $\epsilon=0$. In the nontrivial situation when $\xi \neq 0$, we conclude that $\zeta=\zeta_{0}$, reducing to the previous case.

\section{Two Lemmas}

We give here two preliminary results detailing the extremals in the inequality (1.11), precisely stated in Theorem 1.7.

Lemma 6.1. Let $\alpha \in \bigwedge^{2}\left(\mathbb{R}^{n}\right)$ be represented in the associated basis as

$$
\alpha=a_{1}\left(\xi^{1} \wedge \zeta^{1}\right)+\ldots+a_{k}\left(\xi^{k} \wedge \zeta^{k}\right)
$$

where $k=2,3, \ldots,\left[\frac{n}{2}\right]$ and $a_{1}, \ldots, a_{k}>0$. Suppose $\beta \in \bigwedge^{2 l}\left(\mathbb{R}^{n}\right), l \leq k$, is a nonzero element of the exterior algebra generated by $\left(\xi^{1} \wedge \zeta^{1}\right), \ldots,\left(\xi^{k} \wedge \zeta^{k}\right)$. Then

$$
|\alpha \wedge \beta|^{2} \leq(l+1)\left(1-\frac{l}{k}\right)|\alpha|^{2}|\beta|^{2}
$$

If $1 \leq l<k-1$ then equality occurs only when $a_{1}=\ldots=a_{k}$ and

$$
\beta=b(\underbrace{\alpha \wedge \ldots \wedge \alpha}_{l \text { times }})
$$

for some $b \neq 0$.

Remark. For $l=k-1$ equality takes place for every $\alpha$. In this case the constant in (6.1) is equal to 1 .

Proof. The case $l=k$ being obvious is omitted. We shall consider all ordered $l$-tuples $I=\left(i_{1}, \ldots, i_{l}\right)$ with indices $i_{1}, \ldots, i_{l} \in K=\{1, \ldots, k\}$, and use the notation $\xi^{I}=\xi^{i_{1}} \wedge \ldots \wedge \xi^{i_{l}}, \zeta^{I}=\zeta^{i_{1}} \wedge \ldots \wedge \zeta^{i_{l}}$ for the corresponding exterior monomials. Every $2 l$-covector in the algebra mentioned above can be written as

$$
\beta=\sum_{I \subset K} B_{I} \xi^{I} \wedge \zeta^{I}
$$

where $B_{I} \in \mathbb{R}$. By orthogonality, $|\alpha|^{2}=\sum_{1 \leq i \leq k} a_{i}^{2}$ and $|\beta|^{2}=\sum_{I \subset K} B_{I}^{2}$. We express the wedge product of $\alpha$ and $\beta$ by

$$
\alpha \wedge \beta=\sum_{I \subset K} \sum_{i \notin I}\left(a_{i} B_{I}\right) \xi^{i} \wedge \zeta^{i} \wedge \xi^{I} \wedge \zeta^{I}=\sum_{J \subset K} \sum_{j \in J}\left(a_{j} B_{J \backslash j}\right) \xi^{J} \wedge \zeta^{J}
$$


where the sum runs over all ordered $(l+1)$-tuples $J=\left(j_{0}, j_{1}, \ldots, j_{l}\right)$ whose indices are in $K$. The notation $J \backslash j$ stands for the $l$-tuple obtained from $J$ by deleting an element $j \in J$. By orthogonality,

$$
|\alpha \wedge \beta|^{2}=\sum_{J \subset K}\left(\sum_{j \in J} a_{j} B_{J \backslash j}\right)^{2}=\sum_{J \subset K} \sum_{p, q \in J} a_{p} a_{q} B_{J \backslash p} B_{J \backslash q}
$$

For reason to be clear afterwards we choose a parameter $t=1-\frac{l+1}{k}$, and write

$$
\begin{aligned}
&|\alpha \wedge \beta|^{2}=\frac{1}{2} \sum_{J \subset K} \sum_{p, q \in J}[ t\left(a_{p}^{2} B_{J \backslash p}^{2}+a_{q}^{2} B_{J \backslash q}^{2}\right)+(1-t)\left(a_{p}^{2} B_{J \backslash q}^{2}+a_{q}^{2} B_{J \backslash p}^{2}\right) \\
&\left.-t\left(a_{p} B_{J \backslash p}-a_{q} B_{J \backslash q}\right)^{2}-(1-t)\left(a_{p} B_{J \backslash q}-a_{q} B_{J \backslash p}\right)^{2}\right]
\end{aligned}
$$

Hence

$$
\begin{aligned}
& |\alpha \wedge \beta|^{2}+\frac{1}{2} \sum_{J \subset K} \sum_{p, q \in J}\left[t\left(a_{p} B_{J \backslash p}-a_{q} B_{J \backslash q}\right)^{2}+(1-t)\left(a_{p} B_{J \backslash q}-a_{q} B_{J \backslash p}\right)^{2}\right] \\
& =\sum_{J \subset K}\left[(1+t l) \sum_{p \in J} a_{p}^{2} B_{J \backslash p}^{2}+(1-t) \sum_{\substack{p \neq q \\
p, q \in J}} a_{p}^{2} B_{J \backslash q}^{2}\right] \\
& =(1+t l) \sum_{I \subset K} \sum_{i \in K \backslash I} a_{i}^{2} B_{I}^{2}+(1-t)(k-l) \sum_{I \subset K} \sum_{i \in I} a_{i}^{2} B_{I}^{2} \\
& =(l+1)\left(1-\frac{l}{k}\right) \sum_{I \subset K} \sum_{i \in K} a_{i}^{2} B_{I}^{2}=(l+1)\left(1-\frac{l}{k}\right)|\alpha|^{2}|\beta|^{2}
\end{aligned}
$$

This identity implies inequality (6.1), since $0 \leq t \leq 1$. If $1 \leq l<k-1$ equality occurs if and only if

$$
a_{p} B_{J \backslash p}=a_{q} B_{J \backslash q} \quad \text { and } \quad a_{p} B_{J \backslash q}=a_{q} B_{J \backslash p}
$$

for all ordered (l+1)-tuples $J \subset K$ and all $p, q \in J$. This yields $a_{1}=\ldots=a_{k}$ and $B_{J \backslash p}=B_{J \backslash q}=b$, hence

$$
\beta=b \sum_{I \subset K} \xi^{I} \wedge \zeta^{I}
$$

and formula (6.2) follows. However, for $l=k-1$ we have only the second set of conditions at (6.4) which yields $B_{J \backslash p}=b a_{p}$ for all $p=1, \ldots, k$, where 
$b$ is any real number. This gives formula

$$
\beta=b \sum_{i+I=K} a_{i} \xi^{I} \wedge \zeta^{I}
$$

It is straightforward to see that $\alpha \wedge \beta=|\alpha||\beta| \xi^{K} \wedge \zeta^{K}$.

Next we sharpen Lemma 6.1.

Lemma 6.2. Let $\alpha \in \bigwedge^{2}\left(\mathbb{R}^{n}\right)$ have rank $\leq 2 m$ and $\beta \in \bigwedge^{s}\left(\mathbb{R}^{n}\right)$ with $0 \leq s \leq m-1$. If $2 m=n$ and $s=2 l+1$ then

$$
|\alpha \wedge \beta|^{2} \leq(l+1)\left(1-\frac{l}{m-1}\right)|\alpha|^{2}|\beta|^{2}
$$

Equality occurs if and only if $\operatorname{rank} \alpha=2 m-2$,

$$
\alpha=a\left(\xi^{1} \wedge \zeta^{1}+\ldots+\xi^{m-1} \wedge \zeta^{m-1}\right)
$$

and

$$
\beta=\left(p \xi^{m}+q \zeta^{m}\right) \wedge \underbrace{\alpha \wedge \ldots \wedge \alpha}_{l \text { times }}, \quad p, q \in \mathbb{R}
$$

If $s=2 l$, or $s=2 l+1$ and $2 m<n$, then

$$
|\alpha \wedge \beta|^{2} \leq(l+1)\left(1-\frac{l}{m}\right)|\alpha|^{2}|\beta|^{2}
$$

Equality occurs if and only if

$$
\alpha=a\left(\xi^{1} \wedge \zeta^{1}+\ldots+\xi^{m} \wedge \zeta^{m}\right)
$$

and

$$
\beta=\left(b+p_{m+1} \xi^{m+1}+q_{m+1} \zeta^{m+1}+\ldots\right) \wedge \underbrace{\alpha \wedge \ldots \wedge \alpha}_{l \text { times }}
$$

where $b, p_{m+1}, q_{m+1}, \ldots$ are real numbers.

Proof. We proceed analogously to the proof of Lemma 6.1 by choosing the associated orthonormal basis in $\bigwedge^{1}\left(\mathbb{R}^{n}\right)$, say $\left\{\xi^{1}, \zeta^{1}, \ldots, \xi^{k}, \zeta^{k}, \ldots\right\}$ such that

$$
\alpha=a_{1}\left(\xi^{1} \wedge \zeta^{1}\right)+\ldots+a_{k}\left(\xi^{k} \wedge \zeta^{k}\right)
$$


with positive coefficients. Let $K=\{1,2, \ldots, k\}$. Every $s$-covector can be written as

$$
\beta=\sum_{K \cap M \cap N=\emptyset} \sum_{L \subset K} B_{M N}^{L} \xi^{L} \wedge \zeta^{L} \wedge \xi^{M} \wedge \zeta^{N}
$$

where $M$ and $N$ are ordered subsets of $\{1,2, \ldots, n\}$. The tuples $L, M, N$ are constrained by the condition $2 \# L+\# M+\# N=s=\operatorname{deg} \beta$. In particular,

$$
0 \leq \# L \leq l
$$

It is also required that the coefficients $B_{M N}^{L}$ are different from zero only when $L \cap M=L \cap N=\emptyset$. This requirement involves no loss of generality, while at the same time it quarantees that

$$
|\beta|^{2}=\sum_{K \cap M \cap N=\emptyset} \sum_{L \subset K}\left|B_{M N}^{L}\right|^{2}=\sum_{K \cap M \cap N=\emptyset}\left|\sum_{L \subset K} B_{M N}^{L} \xi^{L} \wedge \zeta^{L}\right|^{2}
$$

We realize that the exterior forms

$$
\beta_{M N}=\sum_{L \subset K} B_{M N}^{L} \xi^{L} \wedge \zeta^{L}
$$

lie in the exterior algebra generated by the 2-covectors $\xi^{i} \wedge \zeta^{i}$ with $i \in K$. The same is true for the wedge products $\alpha \wedge \beta_{M N}$. That is why the terms in the formula

$$
\alpha \wedge \beta=\sum_{K \cap M \cap N=\emptyset}\left(\alpha \wedge \beta_{M N}\right) \wedge \xi^{M} \wedge \zeta^{N}
$$

are also mutually orthogonal. This yields

$$
|\alpha \wedge \beta|^{2}=\sum_{K \cap M \cap N=\emptyset}\left|\alpha \wedge \beta_{M N} \wedge \xi^{M} \wedge \zeta^{N}\right|^{2}
$$

By Lemma 6.1 we have

$$
\begin{aligned}
\left|\alpha \wedge \beta_{M N} \wedge \xi^{M} \wedge \zeta^{N}\right|^{2} & \leq\left|\alpha \wedge \beta_{M N}\right|^{2} \\
& \leq\left(l_{M N}+1\right)\left(1-\frac{l_{M N}}{k}\right)|\alpha|^{2}\left|\beta_{M N}\right|^{2}
\end{aligned}
$$

where $2 l_{M N}=\operatorname{deg} \beta_{M N} \leq \operatorname{deg} \beta=s \leq m-1$. This yields

$$
\left(l_{M N}+1\right)\left(1-\frac{l_{M N}}{k}\right) \leq\left(l_{M N}+1\right)\left(1-\frac{l_{M N}}{m}\right) \leq(l+1)\left(1-\frac{l}{m}\right)
$$


By substituting this into (6.20), in view of (6.19), we obtain

$$
\begin{aligned}
|\alpha \wedge \beta|^{2} & \leq(l+1)\left(1-\frac{l}{m}\right)|\alpha|^{2} \sum_{K \cap M \cap N=\emptyset}\left|\beta_{M N}\right|^{2} \\
& =(l+1)\left(1-\frac{l}{m}\right)|\alpha|^{2}|\beta|^{2}
\end{aligned}
$$

by formula (6.16), completing the proof of inequality (6.10).

Inequality (6.10) is sharp whenever $s$ is even or $2 m<n$. To identify the extremals we must have equality in every step of our calculation. By (6.21) we see that $k=m$ and all numbers $l_{M N}$ are equal to $l$. Recall that we have used Lemma 6.1 for inequality (6.20), where at least one of the $2 l$-covectors $\beta_{M N}$ is nonzero. Accordingly, since $l<s \leq m-1$, we have

$$
\alpha=a\left(\xi^{1} \wedge \zeta^{1}+\ldots+\xi^{m} \wedge \zeta^{m}\right)
$$

and (6.2) gives

$$
\beta_{M N}=b_{M N}(\underbrace{\alpha \wedge \ldots \wedge \alpha}_{l \text { times }}), \quad b_{M N} \in \mathbb{R}
$$

Now, if $\operatorname{deg} \beta=2 l$ we obtain formula (6.12) with $b \in \mathbb{R}$ and $p_{m+1}=$ $q_{m+1}=\ldots=0$. If $\operatorname{deg} \beta=2 l+1$ then the exterior monomials $\xi^{M} \wedge \zeta^{N}$ are actually 1 -covectors, which in view of the first inequality at (6.20) are linear combinations of $\xi^{m+1}, \zeta^{m+1}, \ldots$ Hence (6.12) follows. However, this formula fails when $\operatorname{rank} \alpha=2 m=n$ and $s=2 l+1$, because there is no covector $\xi^{m+1}, \zeta^{m+1}, \ldots$ in reserve for making $\operatorname{deg} \beta=2 l+1$. This case needs handling with somewhat greater care. We shall have formulas (6.8) and (6.9) established once we proof inequality (6.7). To this end, we look closely at the left hand side of (6.20). For every pair $M, N$ the exterior monomial $\xi^{M} \wedge \zeta^{N}$ contains one of the covectors $\xi^{1}, \zeta^{1}, \ldots, \xi^{m}, \zeta^{m}$, as they constitute the complete set of basis elements. On the other hand

$$
\alpha \wedge \xi^{M} \wedge \zeta^{N}=a_{1}\left(\xi^{1} \wedge \zeta^{1} \wedge \xi^{M} \wedge \zeta^{M}\right)+\ldots+a_{k}\left(\xi^{k} \wedge \zeta^{k} \wedge \xi^{M} \wedge \zeta^{M}\right)
$$

where one of the terms vanishes, unless $k<m$. It will not affect the norms in the sum at formula (6.19) if we replace $\alpha$ by

$$
\begin{aligned}
\alpha^{\prime}=a_{1}\left(\xi^{1} \wedge \zeta^{1}\right)+\ldots & +a_{i-1}\left(\xi^{i-1} \wedge \zeta^{i-1}\right) \\
& +a_{i+1}\left(\xi^{i+1} \wedge \zeta^{i+1}\right)+\ldots+a_{m}\left(\xi^{m} \wedge \zeta^{m}\right)
\end{aligned}
$$

with $a_{1}, \ldots, a_{m} \geq 0$, where the missing index $i$ depends on $M, N$; one that belongs to $M \cup N$. 
We next sharpen the inequality (6.20) as

$$
\begin{aligned}
\left|\alpha \wedge \beta_{M N} \wedge \xi^{M} \wedge \zeta^{N}\right|^{2} & =\left|\alpha^{\prime} \wedge \beta_{M N} \wedge \xi^{M} \wedge \zeta^{N}\right|^{2} \leq\left|\alpha^{\prime} \wedge \beta_{M N}\right|^{2} \\
& \leq\left(l_{M N}+1\right)\left(1-\frac{l_{M N}}{m-1}\right)\left|\alpha^{\prime}\right|^{2}\left|\beta_{M N}\right|^{2} \\
& \leq(l+1)\left(1-\frac{l}{m-1}\right)|\alpha|^{2}\left|\beta_{M N}\right|^{2}
\end{aligned}
$$

By reasoning as before we conclude with inequality (6.7). Equality may occur if and only if rank $\alpha=2 m-2$ and $\alpha$ takes the form (6.8). Formula (6.9) is then a consequence of (6.12) with $b=0$ and $m$ replaced by $m-1$.

\section{Concluding remarks}

Having Lemma 6.2 we are now in a position to complete the arguments for the results stated in Introduction.

First we take on stage Theorem 1.6. Let $\alpha \in \bigwedge^{2}\left(\mathbb{R}^{n}\right)$ have rank $2 r$, $1 \leq r \leq\left[\frac{n}{2}\right]$.

Case 1. Assume that $0 \leq s<r$ and apply Lemma 6.2 with $m=r$. If $n=2 r$ and $s$ is odd, say $s=2 l+1$, then by (6.7) we obtain

$$
A_{n, r}^{2}(s)=(l+1)\left(1-\frac{l}{m-1}\right)=\frac{(s+1)(n-s-1)}{2(n-2)}
$$

as in (1.13). If $n=2 r$ and $s$ is even, say $s=2 l$, then by (6.10) we obtain

$$
A_{n, r}^{2}(s)=(l+1)\left(1-\frac{l}{m-1}\right)=\frac{1}{r}\left[\frac{s+2}{2}\right]\left[\frac{2 r-s+1}{2}\right]
$$

as in the first part of formula (1.14). If $n \neq 2 r$ then (6.10) yields

$$
A_{n, r}^{2}(s)=\left(\left[\frac{s}{2}\right]+1\right)\left(1-\frac{1}{r}\left[\frac{s}{2}\right]\right)=\frac{1}{r}\left[\frac{s+2}{2}\right]\left[\frac{2 r-s+1}{2}\right]
$$

which again coincides with the first part of formula (1.14).

Case 2. Assume that $n-r-2<s \leq n-2$. This case is dual to the first one via the symmetry relation $A_{n, r}(s)=A_{n, r}(n-s-2)$ at (1.21). The first formula at (1.14) applies to $n-s-2$ in place of $s$, resulting in the third formula at (1.14). To obtain the full strength of Theorem 1.6 we must examine the third case.

Case 3. Assume that $r \leq s \leq n-r-2$. This needs only slight modification of the proof of Lemma 6.2. Precisely, one should replace the constant 
$\left(l_{M N}+1\right)\left(1-\frac{l_{M N}}{k}\right)$ in $(6.20)$ by $\max (l+1)\left(1-\frac{l}{r}\right)$, where $l$ and $k$ are subjected to the same constrains as in (1.12). This leads us to the middle part of formula (1.14). We leave it to the reader to verify that (1.12) reduces to (1.13) and (1.14) in all cases.

Theorem 1.3 follows by an elementary analysis of formula (1.12). Indeed, the largest value of $A_{n, r}^{2}(s)$, as $r$ varies from 1 to $\left[\frac{n}{2}\right]$, equals

$$
\frac{\left[\frac{n-s}{2}\right]\left[\frac{s+2}{2}\right]}{\left[\frac{n-s}{2}\right]+\left[\frac{s}{2}\right]}=C_{n}^{2}(2, s)
$$

as desired.

Theorems 1.7 and 1.8 follow from the analysis of the extreme cases in the proof of Lemma 6.2.

The last thing we wish to clarify here is the identity (1.5). Suppose $\alpha^{1}, \ldots, \alpha^{k}$ are the unit covectors for which we have equality in (1.3). Thus

$$
\begin{aligned}
{\left[C_{n}\left(l_{1}, \ldots, l_{k}\right)\right]^{2} } & =\left|\alpha^{1} \wedge \cdots \wedge \alpha^{k}\right|^{2}=\left|\left(\alpha^{1} \wedge \cdots \wedge \alpha^{k}\right) \wedge *\left(\alpha^{1} \wedge \cdots \wedge \alpha^{k}\right)\right| \\
& \leq C_{n}\left(l_{1}, \ldots, l_{k}, n-l_{1}-\ldots-l_{k}\right)\left|\alpha^{1}\right| \cdots\left|\alpha^{k}\right|\left|*\left(\alpha^{1} \wedge \cdots \wedge \alpha^{k}\right)\right| \\
& =C_{n}\left(l_{1}, \ldots, l_{k}, n-l_{1}-\ldots-l_{k}\right)\left|\left(\alpha^{1} \wedge \cdots \wedge \alpha^{k}\right)\right| \\
& =C_{n}\left(l_{1}, \ldots, l_{k}, n-l_{1}-\ldots-l_{k}\right) C_{n}\left(l_{1}, \ldots, l_{k}\right)
\end{aligned}
$$

Hence $C_{n}\left(l_{1}, \ldots, l_{k}\right) \leq C_{n}\left(l_{1}, \ldots, l_{k}, n-l_{1}-\ldots-l_{k}\right)$. For the reverse inequality, consider the unit covectors $\beta^{i} \in \Lambda^{l_{i}}\left(\mathbb{R}^{n}\right), i=1, \ldots, k$, and $\beta \in \bigwedge^{n-l_{1}-\ldots-l_{k}}\left(\mathbb{R}^{n}\right)$ such that

$$
C_{n}\left(l_{1}, \ldots, l_{k}, n-l_{1}-\ldots-l_{k}\right)=\left|\beta^{1} \wedge \cdots \wedge \beta^{k} \wedge \beta\right|
$$

By Schwarz inequality (1.2) we obtain

$$
\begin{aligned}
C_{n}\left(l_{1}, \ldots, l_{k}, n-l_{1}-\ldots-l_{k}\right) & \leq\left|\beta^{1} \wedge \cdots \wedge \beta^{k}\right||\beta| \\
& \leq C_{n}\left(l_{1}, \ldots, l_{k}\right)\left|\beta^{1}\right| \cdots\left|\beta^{k}\right||\beta|=C_{n}\left(l_{1}, \ldots, l_{k}\right)
\end{aligned}
$$

Finally, while we don't have proof yet, numerical computations done by S. Perera and T. Holecek at the University of Wisconsin-Superior suggest

Conjecture 7.1. If $n=k l$, with positive integers $k$ and $l$, then

$$
C_{n}(l, l)=\sqrt{2-\frac{2}{k}}
$$


Their investigations are summarized in the preprint [2].

\section{References}

[1] H. Federer, Geometric Measure Theory, Springer, 1969.

[2] T. Holocek, S. Perera, and C. Scott, Improved Estimates for the Exterior Product's Submultiplication Constant, Preprint, 1997.

Tadeusz Iwaniec

Department of Mathematics

Syracuse University, NY 13244

U.S.A.

(E-mail: tiwaniec@mailbox.syr.edu)

Janne Kauhanen

University of Jyväskylä

Department of Mathematics

P.O. Box 35

FIN-40014 Jyväskylän yliopisto

Finland

(E-mail: janne.kauhanen@maths.jyu.fi)

Aleda Kravetz and Chad Scott

Department of Mathematics

Unversity of Wisconsin - Superior

U.S.A.

(E-mails : akravetz@acad.uwsuper.edu ; cscott@facstaff.uwsuper.edu)

(Received : July 2003) 


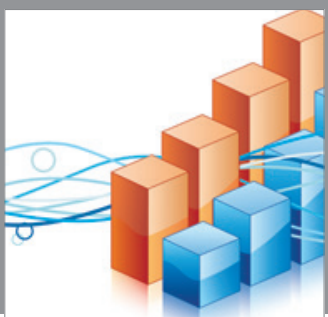

Advances in

Operations Research

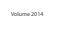

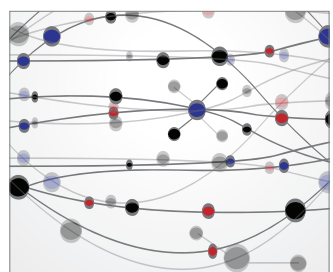

\section{The Scientific} World Journal
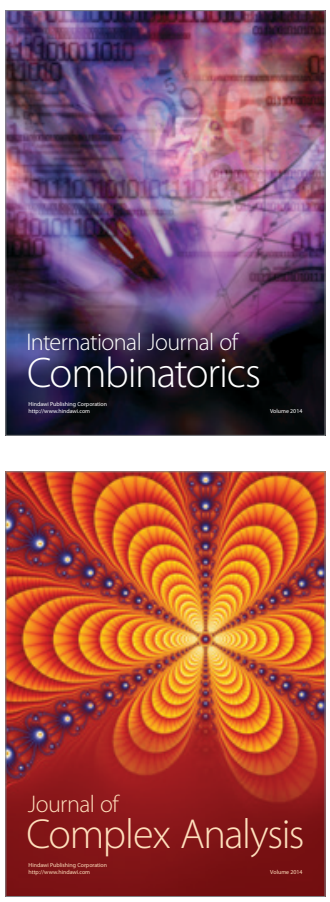

International Journal of

Mathematics and

Mathematical

Sciences
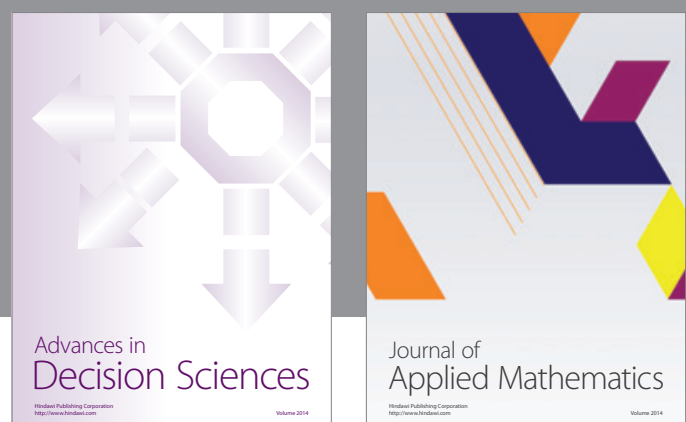

Journal of

Applied Mathematics
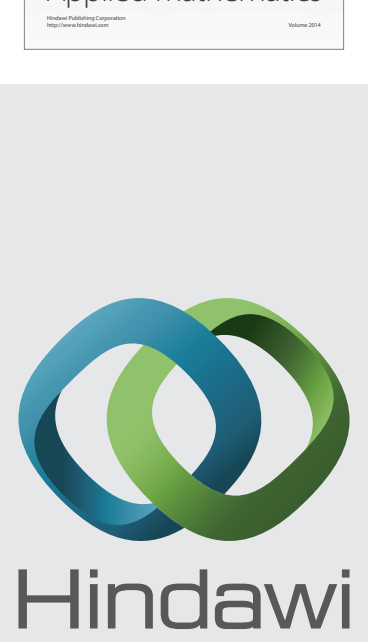

Submit your manuscripts at http://www.hindawi.com
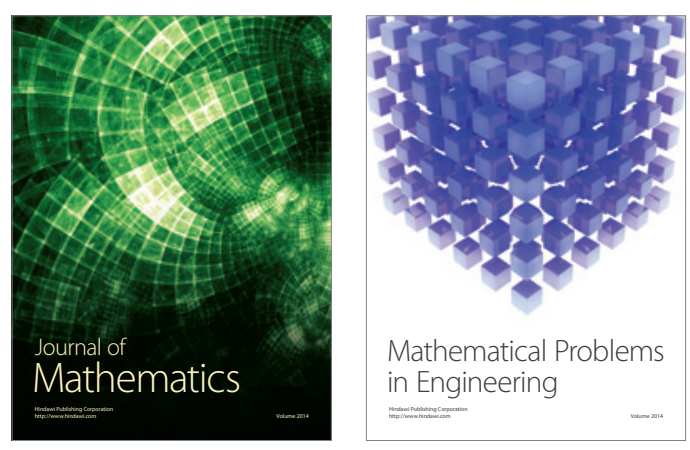

Mathematical Problems in Engineering
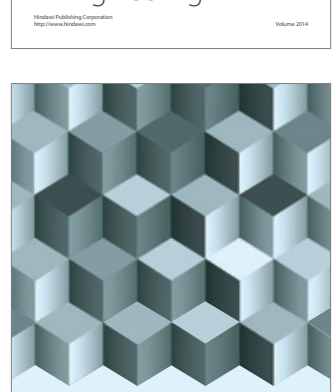

Journal of

Function Spaces
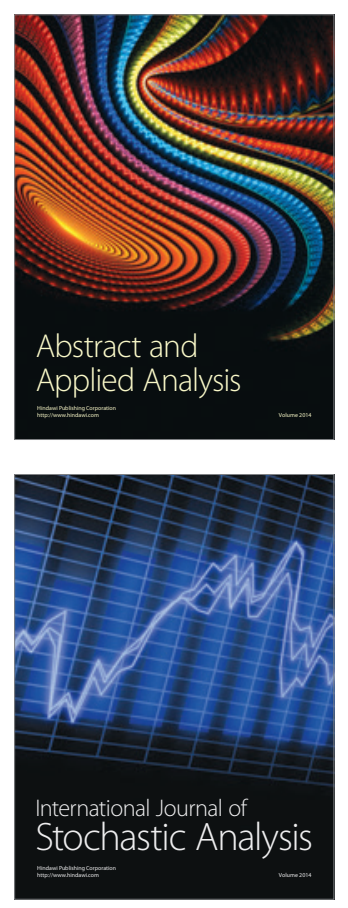

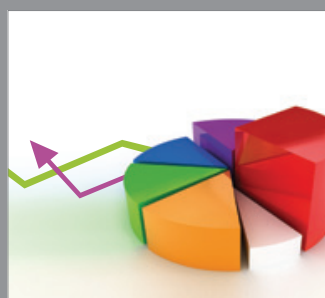

ournal of

Probability and Statistics

Promensencen
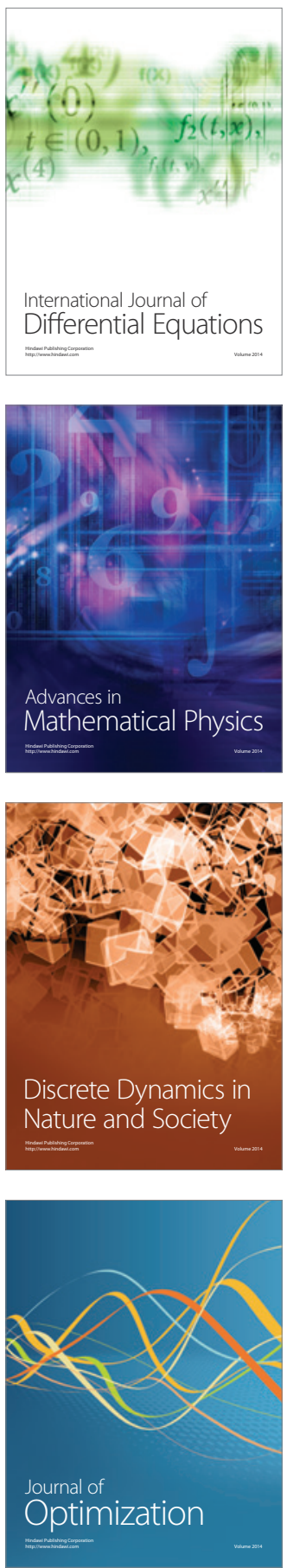